\title{
Adherencia al tratamiento y nivel de control en pacientes asmáticos en un hospital de Lima
}

Rodriguez-Marino J* 1, a; Caballero-García M 1,a; Llanos-Tejada F 1,2,b

\section{RESUMEN}

Objetivo: Describir la asociación entre el control del asma y la adherencia al tratamiento en los pacientes del Hospital Nacional Dos de Mayo en los años 2019-2020.

Materiales y métodos: Se realizó un estudio cuantitativo, descriptivo y retrospectivo, de tipo clínico, durante el periodo 2019-2020, en pacientes asmáticos del Hospital Nacional Dos de Mayo (HNDM) para evaluar la relación entre control del asma y adherencia al tratamiento en los pacientes atendidos en el Consultorio de Asma del Servicio de Neumología. La información fue recolectada de las historias clínicas y los datos registrados fueron los siguientes: test de adherencia a los inhaladores (TAI), test de control de asma (ACT), edad, sexo, tratamiento instaurado, tiempo de enfermedad y la valoración nutricional, a través de la revisión de historias clínicas. El análisis estadístico se realizó con el software IBM SPSS Statistics versión 22, y para determinar la asociación se realizó la prueba estadística X².

Resultados: Se evaluaron 187 pacientes mediante historias clínicas, 84 hombres (44,92 \%) y 103 mujeres (55,08 \%). El promedio de edad fue de 59,42 \pm 15,08 años (solo los mayores de edad fueron considerados en el estudio). Mediante el análisis de los datos de las historias clínicas, se determinó que había 86 pacientes controlados (45,99 \%) y que 48 pacientes eran adherentes (25,67\%). El corticoide inhalado (CSI) fue el tratamiento más usado (50,80 \%). El tipo inconsciente fue la modalidad más frecuente de paciente incumplidor $(64,17 \%)$. El promedio de tiempo de enfermedad, en años, estuvo en $21,78 \pm 16,75$.

Conclusiones: El 75\% de los pacientes asmáticos con adherencia al tratamiento controlan la enfermedad.

Palabras clave: Asma; Control; Adherencia al tratamiento; Adultos (Fuente: DeCS BIREME).

\section{Adherence to treatment and level of control among asthma patients at a Lima hospital}

ABSTRACT

Objective: To describe the association between asthma control and adherence to treatment among patients of the Hospital Nacional Dos de Mayo from 2019 to 2020.

Materials and methods: A quantitative, descriptive and retrospective clinical study was carried out in asthma patients treated at the asthma office of the Pneumology Service from the Hospital Nacional Dos de Mayo (HNDM) from 2019 to 2020 to assess the relationship between asthma control and adherence to treatment. The following data was collected from the medical records: test of adherence to inhalers (TAI) results, asthma control test (ACT) results, age, sex, treatment, disease period and nutritional assessment. The statistical analysis was performed using IBM SPSS Statistics statistical software version 22 and the association between both variables was determined by the chi-square test.

Results: One hundred eighty-seven (187) patients were evaluated through their medical records, out of which 84 were men $(44.92 \%)$ and 103 were women (55.08\%). The mean age was $59.42 \pm 15.08$ years (only adults participated in the study). Data from the medical records determined that 86 patients (45.99\%) had their asthma under control and 48 showed adherence to treatment (25.67\%). Inhaled corticosteroid (ICS) was the most widely used treatment (50.80\%). Irresponsible people were the most frequent noncompliant patients $(64.17 \%)$. The mean disease period was $21.78 \pm 16.75$.

Conclusions: Seventy-five percent (75\%) of asthma patients who adhere to treatment are able to control their condition.

Keywords: Asthma; Control; Treatment adherence and compliance; Adults (Source: MeSH NLM).

1 Universidad de San Martín de Porres, Facultad de Medicina Humana. Lima, Perú.

2 Hospital Nacional Dos de Mayo. Lima, Perú.

a Estudiante de pregrado.

b Médico Neumólogo.

*Autor corresponsal. 


\section{INTRODUCCIÓN}

El asma es una enfermedad obstructiva respiratoria reversible caracterizada por una inflamación crónica de la vía aérea y que se demuestra clínicamente por ataques recurrentes de disnea y sibilancias de severidad variable. Las causas pueden ser factores ambientales, así como las características propias de la persona ${ }^{(1,2)}$.

Desde un punto de vista epidemiológico, el asma es una enfermedad sin una localización geográfica específica; es decir, puede coexistir de forma indiferenciada en todo el mundo. Debido a esta característica, 300 millones de personas padecen de asma en el mundo. En América Latina, su prevalencia alcanza un $17 \%$, con fluctuaciones que oscilan entre 5 y $30 \%$. Esta estadística se ve influenciada por el nivel socioeconómico promedio de cada país. La tasa de mortalidad del asma es baja: 3,73 por cada 100000 personas que sufren la enfermedad ${ }^{(3,4)}$.

En el Perú no se han realizado estudios de prevalencia general de asma en adultos, dado que la mayoría de los estudios en el país se han enfocado en la población pediátrica, debido a que en este grupo etario la enfermedad es más frecuente ${ }^{(5,6)}$.

Entre las causas desencadenantes del evento agudo asmático bronquial tenemos los ejercicios de alto rigor, las reacciones alérgicas o eventos estresores (psicológicos y fisiológicos). Debido a las diversas causas que producen una exacerbación asmática, se le considera una enfermedad de origen multifactorial ${ }^{(7-10)}$.

Es importante precisar que el asma tiene una activación fisiopatológica que produce una liberación y una respuesta desmesurada de la histamina, lo que lleva a una broncoconstricción severa. Además de esta vía, existen otras como la falta de surfactante y la disminución de la presión transpulmonar, las cuales van a alterar de igual forma el equilibrio ventilación/perfusión $(\mathrm{V} / \mathrm{Q})^{(2,11)}$.

Respecto al cumplimiento terapéutico, es necesario mencionar su importancia como parte de la resolución de una enfermedad, es decir, qué tanto el paciente entiende, interioriza y sigue las indicaciones por parte de su médico tratante. Al considerar esta variable, es importante tener en cuenta los factores relacionados a una mala adherencia al tratamiento, tanto exógenos como endógenos, somáticos o fisiológicos. Por otro lado, debemos tener en cuenta que el esquema terapéutico es un eslabón importante para lograr el cumplimiento óptimo ${ }^{(12)}$. El incumplimiento del esquema terapéutico es un problema de salud pública que tiene repercusiones como el incremento del riesgo de exacerbaciones y el aumento de la frecuencia de visitas al médico ${ }^{(12,13)}$.

El control del asma es un importante desafío para la salud pública en el Perú y está directamente relacionado con el grado de adherencia al tratamiento. Si esta falla y ocurre una crisis, se puede dañar la calidad de vida de un paciente; además del costo adicional relacionado con la atención de las exacerbaciones a consecuencia de un mal control de la enfermedad. Por lo expuesto, es necesario estudiar la asociación entre la adherencia al tratamiento y nivel de control del asma.

\section{MATERIALES Y MÉTODOS}

\section{Diseño y población de estudio}

Se realizó un estudio cuantitativo, descriptivo y retrospectivo, de tipo clínico, durante el periodo 2019-2020, en el cual evaluamos la relación entre control del asma y la adherencia al tratamiento en pacientes asmáticos atendidos en el Consultorio de Asma del Servicio de Neumología del Hospital Nacional Dos de Mayo.

El tamaño de muestra fue de 187 datos de pacientes, recopilados a través de historias clínicas, con una potencia del $80 \%$ y un nivel de confianza del $95 \%$.

\section{Variables y mediciones}

En el estudio se incluyeron a los adultos de ambos sexos, de 18 a 90 años, con diagnóstico definitivo de asma y con terapia individualizada previa a la consulta registrada en la historia clínica (pacientes con más de 1 año de seguimiento en Neumología y referidos al Consultorio de Asma). Los pacientes con historia de tabaquismo de 10 o más paquetes consumidos por año y los pacientes asmáticos con comorbilidades fueron excluidos del estudio.

Se recabó la información necesaria para la ficha de recolección de datos mediante la revisión de historias clínicas. Las variables evaluadas fueron las puntuaciones calculadas por parte de los médicos especialistas utilizando el ACT (Asthma Control Test) que evalúa el control adecuado del asma mediante un cuestionario de 5 preguntas con respuestas politómicas que miden la frecuencia para poder determinar el nivel de control (14), y el TAI (Test de adherencia a los inhaladores), que evalúa el cumplimiento del tratamiento teniendo en cuenta los factores externos que puedan influir, así como factores inherentes al paciente. Esta prueba consta de 12 preguntas, 10 dirigidas al paciente y 2 al personal de salud que brinda la atención ${ }^{(15)}$. Además, contamos con otras variables como edad, sexo, tratamiento instaurado, tiempo de enfermedad de cada paciente y diagnóstico nutricional.

\section{Análisis estadístico}

Los resultados obtenidos se analizaron con el software estadístico IBM SPSS Statistics versión 22, en el cual se realizaron las tablas para interpretar los datos obtenidos durante la recolección. Para las variables cuantitativas se determinaron las medidas de tendencia central 
(media, mediana y moda) y de dispersión (desviación estándar y varianza); y para las variables cualitativas se analizó la frecuencia. La prueba de $X^{2}$ fue empleada para medir asociación, los valores $p<0,05$ se consideran significativamente estadísticos.

\section{Consideraciones éticas}

El trabajo fue aprobado por los Comité de Ética del Hospital Nacional Dos de Mayo y por el de la Facultad de Medicina Humana de la Universidad de San Martín de Porres. En este estudio seguimos las normas éticas internacionales y se cumplieron los principios de Helsinki.

\section{RESULTADOS}

Se evaluaron 187 pacientes con asma, en los cuales predominaba el sexo femenino, con 55,08 \%. Además, se encontró una edad promedio de 59,42 $\pm 15,08$ años. El puntaje promedio del ACT fue de 19,34 $\pm 3,17$, asimismo, el $54 \%$ de la población de estudio tenía un asma no controlado. La evaluación con el TAl mostró un promedio de $42,73 \pm 4,48$, y el caso más frecuente fue una mala adherencia $(74,33 \%)$. En cuanto al tratamiento, la terapia más frecuente fue el corticoide inhalado, con un $50,80 \%$. La evaluación nutricional mostró que el 70,59 \% de la población estudiada tuvo sobrepeso y obesidad (promedio de índice de masa corporal $27,33 \pm 4,89$ ). El tipo de incumplidor más frecuente fue el inconsciente $(64,17 \%)$ (Tabla 1$)$.

Tabla 1. Análisis univariado de las características generales de la población de estudio

\begin{tabular}{|c|c|}
\hline Variables & $\mathbf{N}(\%)$ \\
\hline Edad (años) $\mathrm{X} \pm \mathrm{DE}^{*}$ & $59,42 \pm 15,08$ \\
\hline \multicolumn{2}{|l|}{ Sexo } \\
\hline Femenino & $103(55,08 \%)$ \\
\hline Masculino & $84(44,92 \%)$ \\
\hline Tiempo de enfermedad (años) $X \pm D E^{*}$ & $21,78 \pm 16,75$ \\
\hline ACT puntaje $e^{* *} X \pm D E^{*}$ & $19,34 \pm 3,17$ \\
\hline \multicolumn{2}{|l|}{ Tipo de control } \\
\hline - $\quad$ Controlado & $86(45,99 \%)$ \\
\hline - $\quad$ No controlado & $101(54,01 \%)$ \\
\hline TAl puntaje ${ }^{* * *} X \pm D E^{*}$ & $42,73 \pm 4,48$ \\
\hline \multicolumn{2}{|l|}{ Tipo de Adherencia } \\
\hline - $\quad$ Buena adherencia & $6(3,21 \%)$ \\
\hline Adherencia intermedia & $42(22,46 \%)$ \\
\hline - $\quad$ Mala adherencia & $139(74,33 \%)$ \\
\hline \multicolumn{2}{|l|}{ Tipo de incumplidor } \\
\hline - $\quad$ Errático & $62(33,16 \%)$ \\
\hline Deliberado & $5(2,67 \%)$ \\
\hline - Inconsciente & $120(64,17 \%)$ \\
\hline \multicolumn{2}{|l|}{ Tipo de tratamiento } \\
\hline - $\quad \mathrm{SABA}^{* * * *}$ & $30(16,04 \%)$ \\
\hline $\mathrm{CSI}^{* * * * *}$ & $95(50,80 \%)$ \\
\hline - $\quad \mathrm{LABA}+\mathrm{CSI}^{* * * * * *}$ & $62(33,16 \%)$ \\
\hline Peso $(\mathrm{kg}) \mathrm{X} \pm \mathrm{DE} \mathrm{E}^{*}$ & $68,16 \pm 12,89$ \\
\hline Talla $(\mathrm{cm}) \mathrm{X} \pm \mathrm{DE}^{*}$ & $157,92 \pm 9$ \\
\hline Diagnóstico nutricional $\mathrm{X} \pm \mathrm{DE}^{*}$ & $27,33 \pm 4,89$ \\
\hline - $\quad$ Sobrepeso y obesidad & $132(70,59 \%)$ \\
\hline No sobrepeso y no obesidad & $55(29,41 \%)$ \\
\hline
\end{tabular}

* Media \pm desviación estándar

** Asthma control test (Test de control del asma)

*** Test adhesión a los inhaladores

****Short acting beta agonists (Agonista beta de acción corta)
***** Corticosteroid inhaled (Corticoesteroide inhalado)

******Long acting beta agonists (Agonista beta de acción larga) + Corticosteroid inhaled (Corticoesteroide inhalado). 
La tabla 2 muestra que en el grupo de pacientes adherentes (buena adherencia y adherencia intermedia), el $75 \%$ tiene el asma controlado, y que el $64,03 \%$ de los no adherentes tiene asma no controlado (OR: 5,34 y con intervalo de confianza 2,55-11,19).
No se demostró diferencia de acuerdo con el sexo, tipo de tratamiento y diagnóstico nutricional $(p>0,05)$. Respecto a la no adherencia, se evidenciaron los 3 tipos de incumplidor $(p<0,05)$ : los más frecuentes fueron los tipos errático e inconsciente (Tabla 2).

Tabla 2. Análisis bivariado de acuerdo al tipo de adherencia

\begin{tabular}{|c|c|c|c|c|c|c|}
\hline \multicolumn{2}{|c|}{ Características } & \multicolumn{2}{|c|}{ Tipo de adherencia } & \multirow[t]{3}{*}{ Total } & \multirow[t]{3}{*}{ Valor de $p$} & \multirow[t]{3}{*}{ OR } \\
\hline & & \multirow{2}{*}{$\begin{array}{c}\text { Sí }(n=48) \\
n(\%)\end{array}$} & \multirow{2}{*}{$\begin{array}{c}\text { No }(n=139) \\
n(\%)\end{array}$} & & & \\
\hline & & & & & & \\
\hline \multicolumn{7}{|l|}{ Control } \\
\hline & Controlado & $36(75,00)$ & $50(35,97)$ & $86(45,99)$ & 0,000 & 5,34 \\
\hline & No controlado & $12(25,00)$ & $89(64,03)$ & $101(54,01)$ & & $(2,55-11,19)$ \\
\hline \multicolumn{7}{|l|}{ Sexo } \\
\hline & Femenino & $23(47,92)$ & $80(57,55)$ & $103(55,08)$ & 0,247 & \\
\hline & Masculino & $25(52,08)$ & $59(42,45)$ & $84(44,92)$ & & \\
\hline \multicolumn{7}{|l|}{ Tipo de tratamiento } \\
\hline & SABA & $7(14,58)$ & $23(16,55)$ & $30(16,04)$ & 0,908 & \\
\hline & CSI & $24(50,00)$ & $71(51,08)$ & $95(50,80)$ & & \\
\hline & $\mathrm{LABA}+\mathrm{CSI}$ & $17(35,42)$ & $45(32,37)$ & $62(33,16)$ & & \\
\hline \multicolumn{7}{|l|}{ Tipo de incumplidor } \\
\hline & Errático & $0(0,00)$ & $62(44,60)$ & $62(33,16)$ & 0,000 & \\
\hline & Deliberado & $0(0,00)$ & $5(3,60)$ & $5(2,67)$ & & \\
\hline & Inconsciente & $48(100,00)$ & $72(51,80)$ & $120(64,17)$ & & \\
\hline \multicolumn{7}{|l|}{ IMC } \\
\hline & Sobrepeso y obesidad & $33(68,75)$ & $99(71,22)$ & $132(70,59)$ & 0,746 & \\
\hline & No sobrepeso y no obesidad & $15(31,25)$ & $40(28,78)$ & $55(29,41)$ & & \\
\hline
\end{tabular}

\section{DISCUSIÓN}

En el presente estudio encontramos una asociación entre la variable "control del asma" y la variable "adherencia al tratamiento" en los pacientes atendidos en la Unidad de Asma del Servicio de Neumología del Hospital Nacional Dos de Mayo durante el periodo 2019-2020. Otras variables que se consideraron fueron edad, sexo, tiempo de enfermedad, tipo de incumplidor, tipo de tratamiento y diagnóstico nutricional.

El 45,99 \% de nuestros pacientes están controlados, a diferencia del $55 \%$ del estudio de Calvo et al. (España) (16) y del $44 \%$ reportado por Alvear et al. (Chile) ${ }^{(17)}$. Respecto a la forma de establecer el control, este fue realizado mediante el ACT, tanto en las investigaciones mencionadas como en este trabajo; sin embargo, a diferencia de los demás, nuestros datos fueron recolectados de las historias clínicas. La similitud cultural en países latinos quizás sea la causa de la frecuencia de control de asma hallada, sin dejar de considerar el hecho de contar con menor población respecto al estudio realizado en España, el que se aproxima a los 700 pacientes ${ }^{(16)}$.

Recabarren et al. ${ }^{(5)}$ determinaron un promedio ACT de 22,27 por consulta en el programa de asma en pediatría en contraparte con este trabajo, en el cual encontramos, como promedio, un puntaje de ACT de 19,34. Si bien existen diferencias entre ambas investigaciones (grupos etarios), en ambas destaca la ausencia de un control óptimo, determinado por el ACT.

Encontramos un promedio de edad de 59,42 años, similar a Park et al. en Corea ${ }^{(18)}$ (promedio de 57,2 años), pero distinto a los resultados de Calvo et al. en España ${ }^{(16)}$ (45,20 años) y, para finalizar, de Alvear et al. en Chile (17) (54,2 años). Debemos mencionar la estadística de países de distintos continentes para demostrar que la prevalencia de la edad 
promedio de pacientes asmáticos no está determinada por el espacio geográfico, sino por un conjunto de factores de riesgo que influyen en todo el mundo ${ }^{(8)}$.

Con respecto al sexo, el femenino representa más de la mitad de casos en el presente estudio, lo que es similar a reportes de España, Chile y Arabia Saudita ${ }^{(16,17,19)}$. En la niñez, el sexo masculino es el más propenso a desarrollar asma, posteriormente, inicia una disminución gradual conforme el paso de los años, hasta invertir la estadística en la edad adulta, es decir, el sexo femenino se vuelve más propenso que el masculino, debido a que este cuenta con la testosterona que actúa como un factor protector ${ }^{(20)}$.

En cuanto al tratamiento, el corticoide inhalado fue el medicamento más ofertado a nuestros pacientes, a diferencia de lo descrito por González et al. en España (21), donde el plan terapéutico más frecuentemente prescrito fue LABA más corticoides inhalados. Esto podría estar ocasionado por el nivel socioeconómico y la diferencia que existe entre un país europeo con un latinoamericano. Por otro lado, en nuestro estudio se encontró que $15 \%$ de pacientes recibían solo SABA como medicación.

Respecto al estado nutricional, $70 \%$ de los participantes tenían sobrepeso y obesidad, al igual que el estudio de Alvear et al. realizado en Chile ${ }^{(17)}$, que reportó casi un $90 \%$ de pacientes con sobrepeso y obesidad. Se corrobora así que un estado nutricional alterado está ligado al desarrollo de asma, además, esto podría ser una consecuencia de estilos de vida no saludables que, generalmente, son influenciados por el estado educativo y económico de los pacientes.

En relación al tipo de incumplidor, el tipo inconsciente es el paciente que cumple con la terapia, pero no con el horario ni frecuencia y/o el que tiene una mala técnica inhalatoria por desconocimiento (22). Este fue el tipo predominante en nuestro estudio, lo contrario al trabajo de Plaza et al. realizado en España ${ }^{(23)}$, en el cual el tipo de incumplidor más frecuente fue el errático (paciente que olvida tomar su medicación) tanto con el método de autorreporte como por el método electrónico con Nexus 6 (inhalador inteligente). Esta diferencia se explicaría por el tipo de educación que se recibe en Europa y también porque el paciente recibe una mejor explicación sobre el tratamiento por parte del médico tratante.

En cuanto al riesgo que conlleva ser o no adherente al tratamiento, podemos decir, de acuerdo a los resultados, que tener un paciente no adherente implica un riesgo cinco veces mayor a que el paciente esté controlado de su enfermedad.

El estudio se realizó con el objetivo de determinar la asociación entre control y adherencia al tratamiento en los pacientes asmáticos del Hospital Nacional Dos de Mayo durante el periodo 2019-2020. Una de las limitaciones de este trabajo es la pandemia del SARS-CoV-2 (COVID-19), la cual nos impidió ampliar nuestra muestra propuesta y no nos permitió recolectar datos de las historias clínicas en los dos primeros meses del presente año. Otra limitación fue la aplicación indirecta de los test, debido a que los datos obtenidos para la investigación se basaron en lo descrito previamente en las historias clínicas.

En conclusión, el $75 \%$ de pacientes con adherencia al tratamiento tiene asma controlada. Finalmente, recomendamos que se realicen más estudios relacionados a la asociación que existe entre el control de la enfermedad y adherencia al tratamiento en un paciente asmático, debido a que, como hemos descrito previamente, existe un conjunto de intervenciones multifactoriales que pueden influir directamente en el manejo adecuado del asma.

Contribución de los autores: JRM: Concepción y diseño del trabajo, recolección y obtención de datos, análisis e interpretación de resultados, y redacción del manuscrito. MCG: Concepción y diseño del trabajo, recolección y obtención de datos, análisis e interpretación de resultados, y redacción del manuscrito. FLT: Revisión crítica y la aprobación de la versión final del artículo, y asesoría técnica.

Fuentes de financiamiento: Este artículo ha sido financiado por los autores.

Conflicto de interés: Los autores declaran no tener ningún conflicto de interés.

\section{REFERENCIAS BIBLIOGRÁFICAS}

1. Organización Mundial de la Salud. Asma [Internet]. OMS; 2020. Disponible en: https://www. who.int/respiratory/asthma/es/

2. Kudo M, Ishigatsubo Y, Aoki I. Pathology of asthma. Front Microbiol. 2013; 4: 263.

3. Ocampo J, Gaviria R, Sánchez J. Prevalencia del asma en América Latina. Mirada crítica a partir del ISAAC y otros estudios. Rev Alerg Mex. 2017; 64(2): 188-97.

4. Lezana V, Arancibia JC. Consideraciones epidemiológicas del asma en Latinoamérica. Neumol pediátr. 2006; 1(2): 45-8.

5. Recabarren A, Parishuaña E, Martínez N, Esquía G. Evaluación comparativa del nivel de control del asma bronquial en pacientes de 12 a 17 años de edad de EsSalud Arequipa. Horiz Med. 2017; 17(2): 22-9.

6. Munayco CV, Arana J, Torres-Chang J, Saravia L, Soto-Cabezas MG. Prevalencia y factores asociados al asma en niños de 5 a 14 años de un área rural del sur del Perú. Rev Perú Med Exp Salud Publica. 2009; 26(3): 307-13.

7. Santos M, Fonseca M, Álvarez M, Nieto PR, Rocha JF. Asma bronquial. Rev Finlay. 2006; 11(1): 64-9.

8. Martínez Aguilar NE. Etiopatogenia, factores de riesgo y desencadenantes de asma. Neumología y cirugía de tórax. 2009; 68: 98-110.

9. Organización Mundial de la Salud. ¿Qué provoca los ataques de 
asma? [Internet]. OMS; 2019. Disponible en: https://www.who.int/ features/qa/46/es/

10. Moncayo B. Asma: fenotipos, factores de riesgo y desencadenantes [Internet]. Elsevier; 2018. Disponible en: https://www.elsevier. com/es-es/connect/medicina/asma-factores-riesgo

11. Instituto Nacional de Estadística e Informática. Asma [Internet]. Lima; 2012. Disponible en: https://www.inei.gob.pe/media/ MenuRecursivo/publicaciones_digitales/Est/Lib1146/cap04.pdf

12. Ministerio de Salud. Boletín Epidemiológico del Perú 2018 [Internet]. Lima; 2018. Disponible en: http://www.dge.gob.pe/portal/docs/ vigilancia/boletines/2018/18.pdf

13. Salas González LM. Diagnóstico y control del Asma bronquial. Rev Méd Costa Rica Centroam. 2016; 73(618): 185-8.

14. AsthmaControlTest.com. Asthma Control Test [Internet]. 2018. Disponible en: https://www.asthmacontroltest.com/South\%20 America/Peru/es/adult

15. Gemasma. Validación Test de Adherencia a Inhaladores (TAI) [Internet]. 2015. Disponible en: https://www.gemasma.com/ validacion-del-test-de-adherencia-a-los-inhaladores-tai-enpacientes-de-asma-y-epoc/

16. Calvo E, Trigueros JA, López A, Sánchez G. Control del asma en pacientes que acuden a consulta de atención primaria en España (estudio ACTIS). Aten Primaria. 2017; 49(10): 586-92.

17. Alvear G, Figueroa L, Hurtado J, Moyano L. Evaluación del grado de control del asma en un centro de atención primaria: Un estudio descriptivo. Rev Chil Enferm Respir. 2016; 32(2): 68-76.

18. Park HJ, Byun MK, Kim HJ, Ahn CM, Rhee CK, Kim K, et al. Regular follow-up visits reduce the risk for asthma exacerbation requiring admission in Korean adults with asthma. Allergy Asthma Clin Immunol. 2018; 14(1): 29.

19. AL-Jahdali H, Ahmed A, AL-Harbi A, Khan M, Baharoon S, Bin Salih $S$, et al. Improper inhaler technique is associated with poor asthma control and frequent emergency department visits. Allergy Asthma Clin Immunol. 2013; 9(1): 8.

20. Río-Navarro BE, Hidalgo-Castro EM, Sienra-Monge JJL. Asma. Bol Med Hosp Infant Mex. 2009; 66(1): 3-33.

21. González Barcala FJ, De la Fuente-Cid R, Álvarez-Gil R, Tafalla M, Nuevo J, Caamaño-Isorna F. Factores asociados con el control del asma en pacientes de atención primaria en España: el estudio CHAS. Arch Bronconeumol. 2010; 46(7): 358-63.

22. Guía de Usuario para Profesionales Sanitarios. Test de Adhesión a los Inhaladores (TAI) [Internet]. España; 2019. Disponible en: http:// www.taitest.com/docs/Guia_Usuario_TAI_ES.pdf

23. Plaza V, Fernández-Rodríguez C, Melero C, Cosío BG, Entrenas LM, de Llano LP, et al. Validation of the 'Test of the Adherence to Inhalers' (TAI) for Asthma and COPD Patients. J Aerosol Med Pulm Drug Deliv. 2016; 29(2): 142-52.

Correspondencia:

Juan Rodriguez Marino

Dirección: Jirón Soldado Francisco La Rosa Mz. D lote 43 Urbanización Villa Alegre - Santiago de Surco. Lima, Perú. Teléfono: +51989072945

Correo electrónico: juan_rodriguez6@usmp.pe

Recibido: 03 de enero de 2021

Evaluado: 18 de enero de 2021

Aprobado: 11 de febrero de 2021

(c) La revista. Publicado por Universidad de San Martín de Porres, Perú. (c) ${ }^{\text {BY }}$ Licencia de Creative Commons Artículo en acceso abierto bajo términos de Licencia Creative Commons Atribución 4.0 Internacional. (http://creativecommons.org/licenses/by/4.0/)

ORCID iDs

Juan Rodriguez Marino

Melanie Caballero Garcia

Félix Llanos Tejada
(ㄱ) https: / / orcid.org/0000-0002-5562-0827

(1) https: / / orcid.org/0000-0002-6148-6249

(ㄱ) https: / / orcid.org/0000-0003-1834-1287 